\title{
Should We be Routinely Performing Arthroscopic Subacromial Decompression During Rotator Cuff Repair (RCR)?
}

\author{
Sachin Kumar ${ }^{1}$, Rajiv Gogna ${ }^{1}$, Daniel Morris ${ }^{1}$, Amol Tambe ${ }^{1}$
}

\section{Abstract}

Symptomatic rotator cuff tears cause pain and impaired function. Arthroscopic rotator cuff repair is an established treatment when nonoperative management fails. However, debate exists regarding the requirement of concurrent subacromial decompression. This review aims to answer pertinent questions and outline relevant literature in the role of arthroscopic subacromial decompression in rotator cuff repair.

Keywords: Decompression; Cuff; Arthroscopic; Repair.

\section{Introduction}

Shoulder complaints are common; with lifetime prevalence ranging from $6.7-66.7 \%$ [1]. Approximately $70 \%$ is related to rotator cuff pathology [2]. The incidence of rotator cuff tear (RCT) is approximately $70 \%$ of those aged 70 years or above; with $40 \%$ having a 'massive' rotator cuff tear (involvement of two or more tendons) [3]. Many are asymptomatic, but frequently it can reduce quality of life years due to pain and impaired function. Symptomatic RCTs present a significant societal and economic burden; particularly with an aging population and increased labour force participation of the elderly [4]. Markov decision model cost-effectiveness analysis suggests operative treatment of RCTs results in lifetime age-weighted mean total societal saving of $\$ 13,771$ (US) per patient [4]. An acute tear is typically seen following shoulder dislocations or trauma in younger patients [3]. However, theories regarding chronic RCT formation pertain to intrinsic and extrinsic factors.

The intrinsic theory, as proposed by Codman [5], describes tendon degeneration occurring within a zone of hypo-vascularity; typically located $10 \mathrm{~mm}$ from its insertion. Poor regenerative properties, eccentric tensile loading and repetitive micro-instability result in inflammatory changes within the tendon; including tenocyte apoptosis, loss of structural integrity and eventual tearing. Calcific tendonitis may also occur and contribute [6].

Neer's extrinsic theory [7] describes tears occurring due to impingement caused by surrounding structures (acromial spur or tilt, acromioclavicular joint spur or arthritis and an os-acromiale) during normal motion arc which leads to a continuum of impingement, partial and eventual full RCT. Additionally, acromial morphology is a component of the extrinsic theory as proposed by Bigliani [8] in which they describe an initiating factor leading to rotator cuff dysfunction and eventual tearing. Consequently, it has been advocated that acromioplasty is an integral part of RCT treatment. Originally classified on outlet view radiographs, described acromion morphology includes flat, curved and hooked with prevalence of $12 \%, 56 \%$ and $29 \%$ respectively. A hooked and curved acromion has been associated with RCT.

Operative treatment is indicated in symptomatic patients when nonoperative management fails. In the absence of joint arthrosis, arthroscopic RCR, if possible, is well established treatment, and this is one of the most frequently performed shoulder surgeries [9]. If successful, improved biomechanics, pain and function can be expected. However, debate exists regarding the requirement of subacromial decompression during rotator cuff repair. This review aims to discuss the role of subacromial decompression in rotator cuff repair and current literature related to the topic.

What is the Current Role of Arthroscopic Subacromial Decompression / Acromioplasty (ASD)?

It is important to clarify that the term 'arthroscopic subacromial decompression' can encompass a variety of components depending on individual surgeon preferences and patient anatomy. Subacromial bursal debridement is an essential part of rotator cuff repair. The bursa has been shown to contain an increased amount of substance $P$, which correlates with pain from primary afferent nerves; hence bursectomy has been proposed to reduce cuff-related shoulder pain [10]. Although bursectomy is well established during decompression, there can be variations in the extent of bone removal, whether the coracoacromial ligament is released, acromioclavicular joint excision, co-planing or

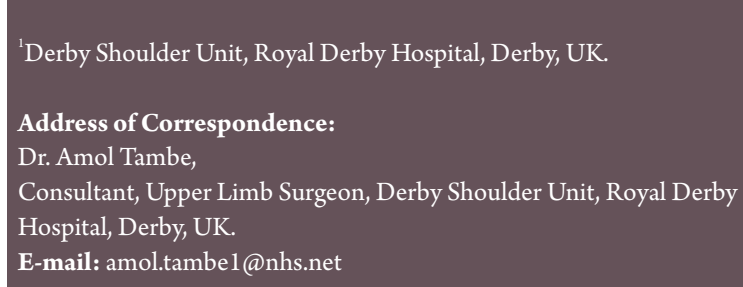

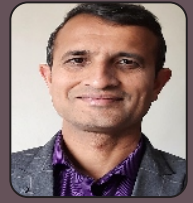

Dr. Sachin Kumar

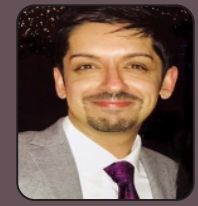

Dr. Rajiv Gogna

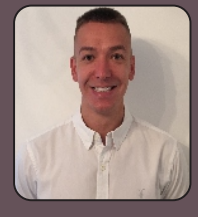

Dr. Daniel Morris

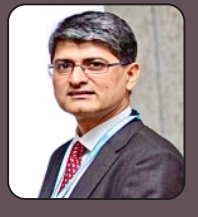

Dr. Amol Tambe

Submitted: 28 March 2021; Reviewed: 21 April 2021; Accepted: 01 May 2021; Published: 10 June 2021

Asian Journal of Arthroscopy | ISSN 2456-1169| Available on www.asianarthroscopy.com | DOI:10.13107/aja.2021.v06i01.025 |

This is an open access journal, and articles are distributed under the terms of the Creative Commons Attribution Non-Commercial-Share Alike 4.0 License (http://creativecommons.org/licenses/by-nc-sa/4.0) which allows others to remix, tweak, and build upon the work non-commercially as long as appropriate credit is given and the new creation are licensed under the identical terms. 
removal of prominent osteophytes. These factors all need to be taken into account, as it remains unclear which component of the decompression, if any, provides the greatest benefit to patients.

This is the reason why arthroscopic subacromial decompression has recently been under increased scrutiny, with several studies questioning its proposed benefits. One of the largest multicentre, randomised controlled trials (C-SAW) [11] compared arthroscopic subacromial decompression versus diagnostic arthroscopy alone versus physiotherapy. At six-month follow-up, they found that the surgical groups had better outcomes for shoulder pain and function but this difference was not clinically important. Interestingly, decompression appeared to offer no additional benefit when compared to diagnostic arthroscopy alone, and the authors suggested the placebo effect of surgery may have a role. Limitations of the study include the high levels of non-compliance between groups as well as the short follow-up period. Since its publication in 2018, the role of decompression has been questioned in all aspects of shoulder surgery, including arthroscopic rotator cuff repair.

\section{What are The Perceived Benefits of Acromioplasty During Rotator} Cuff Repair?

Neer's extrinsic theory of cuff degeneration led to subacromial decompression, (consisting of bursectomy, anteroinferior acromioplasty and release of the coracoacromial ligament), being performed routinely as part of rotator cuff repair surgery. Decompression increases the height of the subacromial space, relieving symptoms of impingement and reducing the risk of attrition to the repaired tendon, with several studies reporting satisfactory results when combined with rotator cuff repair [12]. Acromioplasty was originally thought to be particularly beneficial for patients with type two (curved) or type three (hooked) acromial morphologies.

Furthermore, acromioplasty can provide surgeons with more working space and improved arthroscopic visualisation intraoperatively, as well as altering the vector of deltoid action; thereby reducing strain upon the repaired cuff [13]. Potential biological benefits include the release of growth factors and bone-marrow derived cells from the bleeding cancellous under surface of the acromion. Subacromial fluid samples following decompression have shown elevated concentrations of growth factors and bone-specific matrix metalloproteinases which may improve cuffhealing $[14,15]$.

During arthroscopic rotator cuff repair, the bony decompression can be performed prior to the cuff repair to remove sharp spurs and increase working space, however this may result in bleeding and obscured visualisation. Alternatively, some surgeons advocate performing the cuff repair first before shoulder swelling makes it an increasingly difficult operation.

\section{Does Acromial Morphology Affect Decision to Proceed to} Acromioplasty?

This has remained a quandary and no study had explored this question primarily. However, two RCTs reported the 2-year follow-up of postoperative ASES score of patients with various types (1-3) of acromion $[16,17]$. Their results were compiled by Song et al $[18]$ in a metaanalysis where they found no difference in outcomes between arthroscopic RCR with or without acromioplasty. Interestingly, the majority of the patients in both studies had a type 2 (curved) acromion with far fewer patients having type 1 or type 3 acromial morphology
$[16,17]$. Both studies did not stratify patients according to acromion type, undertake power calculations which could have accounted for subgroup treatment effects or define subgroups based on prerandomization patient characteristics. There is no level I evidence to prove for or against ASD when it comes to acromion morphology.

Has Decompression Been Shown to Reduce Post-Operative Pain? Two studies collected data for post-op VAS scores [17, 19]. Their results were meta-analysed by Song et al [18] and found no significant difference in scores between the two groups. Shin et al [19] found relatively higher VAS in a group of patients undergoing ASD at 3 months and 6 months but it did not reach statistical significance. Unfortunately, not all the studies looked at the post-operative VAS scores, only Shin et al looked at 3 months post op. On the other hand, Abrams et al [17] started collecting data for VAS scores at 6 months post op. There is not enough evidence to compare pain scores between groups with or without acromioplasty. Therefore, the question, whether there is any improvement in pain scores for patients undergoing ASD with RCR in the early post-op period, still remains unanswered.

Is There A Difference in Functional Scores Between the Two Interventions?

This was the primary outcome measure for all six RCTs undertaken on this topic $[6,12,16,17,19,20]$. They all found no difference in the various outcome measures studied. Cheng et al [21] conducted a metaanalysis and included all of these RCTs and one cohort study. They did not find any clinically significant difference in reported outcomes between patients having RCR with or without ASD. Though they found a statistically significant difference in the ASES score (in favour of the acromioplasty group), this difference was very small with a mean of 2.94 (range 0.39-5.48). The clinical value of this difference is questionable as minimal clinically important difference (MCID) for ASES should be in the range of 9-26.9 according to a systematic review conducted by Dabije et al [22].

None the less, Cheng et al did not find any difference in other outcome measures (UCLA, SST, Constant) between the two groups. It will be worth mentioning that this review had good number of patients $(\mathrm{n}=$ 651). Therefore, there is level 1 evidence to support the notion that there is no difference in functional outcomes between groups undergoing RCR with or without ASD. However, all these RCTs had short follow up between 15.6 and 35 months $[6,12,16,17,19,20]$. Since there are no RCTs with longer term follow-up, it is difficult to confirm if there will be a true difference between the two groups over time.

\section{Does ASD Influence the Rate of Re-Operation or Re-tear?}

Re-operation rates were explored by four studies $[16,17,19,20]$. In the group having acromioplasty, 4 patients had capsular release in two studies $[19,20]$ and 12 patients underwent revision RCR in three studies $[17,19,20]$. In the group having RCR without acromioplasty, three $[16,17,20]$ studies reported arthroscopic capsular release in 4 patients, and four studies reported revision RCR in 19 patients $[16,17$, $19,20]$. Cheng et al meta-analyzed these results and did not find any statistically significant difference in re-operation rates between the two groups. Although the re-operation rate reported by two studies $[16,20]$ did not reach statistical significance between the two groups, 5 of the 10 
revision surgeries in these studies were in patients with a type 3 acromion. Therefore, stratification of patients according to acromion type is required to state the true impact of acromion shape on the re-tear rate leading to revision $\mathrm{RCR}$.

Shin et al [19] also did not find any difference in failure rate for rotator cuff healing according to acromion shape in the RCR group without acromioplasty. However, the majority of patients in this study had type 2 acromial morphology, followed by type 1 and type 3 . There is a need for more high-quality studies with post-operative imaging to establish whether there is truly any significant difference in the tear rate between the groups with or without ASD. Without stratifying patients according to acromion type, it would be wrong to conclude that acromion type had no effects on re-tear rates. This has not been addressed in any study thus far.

Are There Any Effects on Patient Satisfaction, Recovery and Return to Sports?

No author reported patient satisfaction as such; however, all 6RCTs did not find any differences in the functional outcomes between both groups $[6,12,16,17,19,20]$. Functional outcomes can be considered as proxy for patient satisfaction as they are based on objective questions answered by patients and it did not differ between patients having RCR with or without ASD. No study reported any outcomes on the length of recovery, return to work or return to sports. These very important questions also need to be addressed in future studies.

\section{Are There Any Problems Associated with Subacromial Decompression During Cuff Repair?}

Potential disadvantages of routinely performing subacromial decompression during arthroscopic rotator cuff repair include violation of the soft tissue envelope resulting in intra-operative swelling, as well as the formation of adhesions and scarring between the raw exposed bone on the acromial under surface and the tendon repair, which can subsequently restrict range of motion, smoothness and comfort. Traditionally, subacromial decompression incorporated release of the coracoacromial ligament. However, the importance of preserving the integrity of coracoacromial arch for maintenance of static and dynamic stability of the glenohumeral joint has been emphasised by Codman [5]. In failed or irreparable rotator cuff tears, releasing the coracoacromial ligament can result in antero-superior glenohumeral joint instability.

If there is excessive bony resection, deltoid insertional pain and weakening due to detachment of its anterior fibres can result. The risk of avulsion of the deltoid origin or post-operative acromial fracture though small, will result in pain and morbidity. This may have potential long-term implications as fracture non-union and deltoid dysfunction may impact the ability to proceed to a reverse shoulder arthroplasty, especially in patients with failed or irreparable rotator cuff tears. Consequently, proponents of the intrinsic theory suggest avoiding subacromial decompression or solely performing subacromial bursectomy.

\section{Senior Authors Preference}

The senior author takes a pragmatic approach. The focus is to undertake a thorough bursectomy and subacromial debridement to outline the RCT and proceed to arthroscopic repair without undertaking bony acromial resection. We undertake our repairs in the beach chair position. Single and double row techniques are undertaken on a case by case basis. A CA ligament release is undertaken only if essential and to improve visualisation if presented with a constricted view. Once the repair is completed, rotation of the proximal humerus will give an indication of physical impingement between the repaired area on the cuff footprint and the acromion. Should this be the case, bony resection is undertaken to achieve impingement free movements. In the absence of specific contact from the acromion onto the repaired area, the senior author will use a shaver to abrade the bony undersurface of the acromion to augment the crimson duvet effect on the repaired cufffootprint.

\section{Conclusion}

In summary, there are certainly theoretical benefits of performing acromioplasty during arthroscopic rotator cuff repair, such as decreasing impingement and wear on the repaired cuff, creating more working space to improve visualisation and the biological effects of marrow derived cells and mediators to improve healing response. However, these proposed benefits should be weighed up against the potential problems highlighted above.

Furthermore, evidence so far has failed to show significant differences in functional outcomes, pain or re-operation rates between the two groups. Most studies do not include imaging data to assess cuff healing and often outcome measures can be insensitive to capture the theoretical benefits of decompression. Future research with longer follow-up is required with sufficient power to determine the relationship between acromial morphology, acromioplasty and surgical outcomes of arthroscopic rotator cuff repair.

\section{References}

1. Luime J, Koes B, Hendriksen I, Burdorf A, Verhagen A, Miedema H, et al. Prevalence and incidence of shoulder pain in the general population; $a$ systematicreview. Scand J Rheumatol.2004;33(2):73-81.

2. Van der Windt D, Koes B, Boeke A. Shoulder disorders in general practice: prognosticindicators of outcome. BrJGen Pr. 1996;46(410):519-23.

3. Micallef J, Pandya J, Low AK. Management of rotator cuff tears in the elderly population. Maturitas [Internet].2019 May;123:9-14.

4. Mather RC, Koenig L, Acevedo D, Dall TM, Gallo P, Romeo A, et al. The Societal and Economic Value of Rotator Cuff Repair. J Bone Jt Surg [Internet].2013 Nov;95(22):1993-2000.
5. Codman E. The Shoulder: Rupture of the supraspinatus tendon and other lesions in or about the subacromial bursa. Boston: Thomas Todd Co.; 1934. 1869-1940p.

6. Gartsman GM, O'Connor DP. Arthroscopic rotator cuff repair with and without arthroscopic subacromial decompression: a prospective, randomized study of one-year outcomes. J Shoulder Elb Surg [Internet]. 2004Jul;13(4):424-6.

7. Neer C, Poppen N. Supraspinatus outlet. Orthop Trans. 1987;(11):234.

8. Bigliani L, Morrison D, April E. The morphology of the acromion and its relationship to rotator cufftears. Orthop Trans. 1986; (10):228. 
9. Sela Y, Eshed I, Shapira S, Oran A, Vogel G, Herman A, et al. Rotator cuff tears: correlation between geometric tear patterns on MRI and arthroscopy and pre- and postoperative clinical findings. Acta radiol [Internet]. 2015 $\mathrm{Feb}$;56(2):182-9.

10. Gotoh M, Hamada K, Yamakawa H, Inoue A, Fukuda H. Increased substance $P$ in subacromial bursa and shoulder pain in rotator cuff diseases. $J$ Orthop Res [Internet].1998 Sep;16(5):618-21.

11. Beard DJ, Rees JL, Cook JA, Rombach I, Cooper C, Merritt N, et al. Arthroscopic subacromial decompression for subacromial shoulder pain (CSAW): a multicentre, pragmatic, parallel group, placebo-controlled, three-group, randomised surgical trial. Lancet [Internet]. 2018 Jan;391(10118):329-38.

12. Milano G, Grasso A, Salvatore M, Zarelli D, Deriu L, Fabbriciani C. Arthroscopic Rotator Cuff Repair With and Without Subacromial Decompression: A Prospective Randomized Study. Arthrosc J Arthrosc RelatSurg [Internet]. 2007Jan;23(1):81-8.

13. Xue G, Chahal K, Lim T, Hu S, Li S, Liu J. Titanium mini locking plate with trans-osseous sutures for the treatment of humeral greater tuberosity fracture osteosynthesis versus PHILOS: a retrospective view. Int Orthop. 2018;42(10):2467-73.

14. Galliera E, Randelli P, Dogliotti G, Dozio E, Colombini A, Lombardi G, et al. Matrix metalloproteases MMP-2 and MMP-9: Are they early biomarkers of bone remodelling and healing after arthroscopic acromioplasty? Injury [Internet].2010 Nov;41(11):1204-7.

15. Randelli P, Margheritini F, Cabitza P, Dogliotti G, Corsi MM. Release of growth factors after arthroscopic acromioplasty. Knee Surgery, Sport Traumatol Arthrosc [Internet]. 2009 Jan 31;17(1):98-101.
16. MacDonald P, McRae S, Leiter J, Mascarenhas R, Lapner P. Arthroscopic Rotator Cuff Repair with and without Acromioplasty in the Treatment of Full-Thickness Rotator Cuff Tears. J Bone Jt Surgery-American Vol [Internet].2011 Nov;93(21):1953-60.

17. Abrams GD, Gupta AK, Hussey KE, Tetteh ES, Karas V, Bach BR, et al. Arthroscopic Repair of Full-Thickness Rotator Cuff Tears With and Without Acromioplasty. Am J Sports Med [Internet]. 2014 Jun 14;42(6):1296-303.

18. Song L, Miao L, Zhang P, Wang W-L. Does concomitant acromioplasty facilitate arthroscopic repair of full-thickness rotator cuff tears? A metaanalysis with trial sequential analysis of randomized controlled trials. Springerplus [Internet].2016 Dec 21;5(1):685.

19. Shin S-J, Oh JH, Chung SW, Song MH. The Efficacy of Acromioplasty in the Arthroscopic Repair of Small- to Medium-Sized Rotator Cuff Tears Without Acromial Spur: Prospective Comparative Study. Arthrosc J Arthrosc RelatSurg [Internet].2012 May;28(5):628-35.

20. Tetteh E, Hussey KE, Abrams GD, Gupta AK, Dhawan A, Karas V, et al. A Prospective Randomized Trial of Functional Outcomes Following Rotator Cuff Repair With and Without Acromioplasty. Orthop J Sport Med [Internet].2013 Sep;1(4_suppl):2325967113S0010.

21. Cheng C, Chen B, Xu H, Zhang Z, Xu W. Efficacy of concomitant acromioplasty in the treatment of rotator cuff tears: A systematic review and meta-analysis. van der Linde JA, editor. PLoS One [Internet]. 2018 Nov 15;13(11):e0207306.

22. Dabija DI, Jain NB. Minimal Clinically Important Difference of Shoulder Outcome Measures and Diagnoses. Am J Phys Med Rehabil [Internet]. 2019Aug;98(8):671-6.

Declaration of patient consent: The authors certify that they have obtained all appropriate patient consent forms. In the form, the patient has given his consent for his images and other clinical information to be reported in the Journal. The patient understands that his name and initials will not be published, and due efforts will be made to conceal his identity, but anonymity cannot be guaranteed.

\section{Conflict of interest: Nil Source of support: None}

\section{How to Cite this Article}

Kumar S, Gogna R, Morris D, Tambe A $\mid$ Should We be Routinely Performing Arthroscopic Subacromial Decompression During Rotator Cuff Repair (RCR)?|Asian Journal of Arthroscopy | January-June 2021; 6(1):48-51. 